Questions vives

\section{Questions Vives}

Recherches en éducation

Vol. $6 n^{\circ} 12$ | 2009

Evaluer les enseignants et les formateurs. Comment ? Pourquoi ? Pour quoi ?

\title{
Vers quelle évaluation de la réflexivité en contexte de formation initiale des enseignants?
}

\section{Antoine Derobertmasure et Arnaud Dehon}

\section{(2) OpenEdition}

Journals

Édition électronique

URL : http://journals.openedition.org/questionsvives/376

DOI : 10.4000/questionsvives.376

ISBN : 978-2-8218-1084-6

ISSN : $1775-433 \mathrm{X}$

Éditeur

Université Aix-Marseille (AMU)

Édition imprimée

Date de publication : 6 juin 2009

Pagination : $29-44$

ISBN : 978-2-912643-36-0

ISSN : 1635-4079

\section{Référence électronique}

Antoine Derobertmasure et Arnaud Dehon, « Vers quelle évaluation de la réflexivité en contexte de formation initiale des enseignants? », Questions Vives [En ligne], Vol. 6 n¹ $^{\circ} 2$ | 2009, mis en ligne le 01 janvier 2011, consulté le 01 mai 2019. URL : http://journals.openedition.org/questionsvives/376 ; DOI 10.4000/questionsvives.376

\section{(c) (i) (9)}

Questions Vives est mis à disposition selon les termes de la licence Creative Commons Attribution Pas d'Utilisation Commerciale - Pas de Modification 4.0 International. 


\title{
Vers quelle évaluation de la réflexivité en contexte de formation initiale des enseignants?
}

\author{
Antoine Derobertmasure, Arnaud Dehon ${ }^{1}$
}

Résumé:En Communauté française de Belgique, la formation des enseignants de l'enseignement secondaire supérieur (niveau lycée en France) est définie par un référentiel de 13 compétences (Décret de 2001 définissant la formation initiale des agrégés de l'enseignement secondaire supérieur). Parmi ces 13 compétences à développer, l'une d'elles met particulièrement l'accent sur la nécessité de développer, dès la formation initiale, la posture réflexive des futurs enseignants. Autant cette notion apparaît centrale du point de vue des décideurs politiques et de la littérature scientifique, autant l'opérationnalisation de cette dimension réflexive au sein de la formation initiale pose problème aux formateurs. Cette difficulté concerne d'une part la définition de la notion de réflexivité et des dispositifs pédagogiques pouvant être mis en place afin de la favoriser et, d'autre part, l'évaluation pouvant être faite de cette dimension. C'est à ces deux difficultés que l'article entend répondre.

Mots-clés: formation d'enseignants, évaluation, réflexivité, analyse de contenu, logiciels d'analyse de contenu.

Abstract: In the French Belgium Community, secondary teacher education is based on 13 skills presented in a decree defining teacher education. Among these 13 skills, one of them focuses on the primary importance of developing student teachers' reflectivity during their training. Although this notion seems to be important from a theoretical and political point of view its inclusion into the training of teachers is difficult. This difficulty is related to the definition of the understanding and to the assessment of this dimension. This contribution tries to answer those questions

Keywords: teacher education, measures, reflectivity, content analysis, content analysis software.

\footnotetext{
1 Chercheurs en Sciences de l'Education - Université de Mons - Institut d'Administration Scolaire (INAS) - Service de Méthodologie et Formation.
} 


\section{Vers quelle évaluation de la réflexivité en contexte de formation initiale des enseignants?}

\section{Contexte}

Les dispositifs de formation initiale des enseignants doivent répondre à un souci de professionnalisation (Lang, 1996 ; Gervais \& Lepage, 2000 ; Martin, Garant, Gervais \& SaintJarre, 2000 ; Maroy, 2001 ; Paquay, 2005). Bien que recommandée dans la littérature et par les pouvoirs politiques, la notion de professionnalisation est encore difficilement appréhendable dans une optique d'évaluation. Rendre une formation professionnalisante est d'ailleurs davantage associé à la finalité de formation plutôt qu'à une démarche concrète à mettre en place. II existe d'ailleurs peu d'indicateurs objectifs qui permettent d'évaluer directement le niveau de professionnalisation de dispositifs d'enseignement.

Liées à la notion de professionnalité, celles de réflexivité - et de praticien réflexif (Schön, 1983 ; Paquay \& Wagner, 2001) - sont également incontournables (Van Manen, 1977; Kirby \& Teddlie, 1989 ; Kirby \& Paradise, 1992 ; Tochon, 1993 ; Schön, 1994 ; Durand, 1996 ; Lang, 1996 ; Maroy, 2001 ; Boutet, 2004 ; Scheepers, 2005 ; Peters, Chevrier, Leblanc, Fortin \& Malette, 2005 ; Donnay \& Charlier, 2006 ; Paquay, Altet, Charlier \& Perrenoud, 2006 ; De Cock, 2007). Désignée comme une facette de la professionnalité - " un praticien qui conceptualise mobilise un aspect de la pensée réflexive et par conséquent un aspect de la professionnalité" (Jorro, 2005, 45) - la réflexivité renvoie à la capacité de réfléchir délibérément (Peters et al., 2005 ; De Cock, 2007 ; Dewey, 1933 cité par De Cock, 2007) sur ses propres pratiques (Perrenoud, 2001) en vue de résoudre des problèmes (Hatton \& Smith, 1995), c'est-à-dire en vue d'améliorer ses pratiques (Tochon, 1993). Se distinguant de l'adjectif « réfléchi » qui décrit un état plutôt qu'un processus, la réflexivité est donc englobée dans le modèle de la professionnalité et constitue, pour Durant (1996) et Maubant (2007), le passage obligé entre le statut de métier et celui de profession. Les dispositifs professionnalisants doivent donc valoriser l'action professionnelle et la réflexion sur celle-ci tout en confrontant les futurs enseignants à des savoirs scientifiques "à " enseigner et " pour » enseigner.

Dans cette perspective, la Communauté française de Belgique a élaboré un décret (Décret du 8 février 2001; MB 22/02/2001) régissant la formation initiale des enseignants de l'enseignement secondaire supérieur (niveau lycée) mettant l'accent sur le développement de 13 compétences professionnelles et sur la construction d'une identité professionnelle ${ }^{2}$ forte (Paquay, 2005). Au sein de l'Institut d'Administration scolaire de l'Université de Mons's en

2 L'identité professionnelle comprend « l'ensemble des composantes représentationnelles, opératoires et affectives produites par l'histoire particulière d'un individu et qu'il est susceptible de mobiliser à un moment donné dans une pratique" (Barbier, 1996 cité par Beckers, Paquay, Coupremanne \& Scheepers, 2002).

${ }^{3}$ En Belgique, les universités sont les seuls opérateurs à pouvoir décerner les titres d'agrégés de l'enseignement secondaire supérieur et ce, pour les disciplines dont elles délivrent le diplôme de master. 
Evaluer les enseignants et les formateurs

Comment, pourquoi, pour quoi ?

charge de la formation pratique des futurs agrégés ${ }^{4}$ (enseignants niveau "lycée »), un dispositif a été mis en place pour répondre à ces prérogatives. Basé sur une alternance entre théorie et pratique (Perrenoud, 2001; Wittorski, 2007), le dispositif vise le développement de trois des 13 compétences du référentiel : développer les compétences relationnelles liées aux exigences de la profession; planifier, gérer et évaluer des situations d'apprentissage et, finalement, porter un regard réflexif sur sa pratique.

En pratique, le dispositif se compose d'un séminaire d'analyse des pratiques (10h), d'un stage d'enseignement (40h) et d'un stage préparatoire à l'enseignement (30h). Dans le but de proposer des situations d'interaction avec les pairs, de planifier ces interactions et de réfléchir sur celles-ci, quatre types d'activités ont finalement été retenus: la vidéo-formation et le micro-enseignement (Wagner, 1998; Paquay, Altet, Charlier \& Perrenoud, 2006), une adaptation de l'autoconfrontation simple (Faïta \& Vieira, 2003; Amigues, Faïta \& Saujat, 2004 ; Yvon \& Garon, 2006) et la réalisation d'un écrit de type réflexif (Jorro, 2005; Scheepers, 2005). Ainsi, après un cours introductif et l'explicitation des objectifs de formation, les enseignants en formation initiale préparent, en duo, deux leçons. Ces séances sont entièrement filmées et certains étudiants jouent le rôle d'élèves ou d'observateurs. La séance terminée, chaque enseignant en formation reçoit une copie vidéo de sa leçon, les grilles d'observation et les commentaires de son binôme. Ensuite, une séance d'échanges entre les enseignants en formation permet de nourrir la réflexion par l'interaction avec les pairs et le partage des expériences. Finalement, avant l'étape conclusive, chaque enseignant en formation expose une préparation de leçon durant un temps imparti. Le but est de cerner les étapes essentielles d'une démarche pédagogique, de synthétiser les idées et de les exposer de manière à être comprises de tous. L'ensemble de ces étapes doit favoriser la réflexion qui est formalisée dans un rapport réflexif remis avant la période de stage dans les écoles.

\section{Problématique et objet de l'étude}

Le dispositif mis en place vise le développement de trois compétences. Afin d'évaluer le dispositif, il est nécessaire de s'interroger sur la mesure selon laquelle celui-ci parvient à atteindre les objectifs qui lui sont assignés. Cet article porte sur l'évaluation de l'atteinte du développement de l'une des trois compétences, c'est-à-dire, celle de " porter un regard réflexif sur sa pratique». La mesure de la réflexivité ne peut être obtenue de manière "directe ", c'est-à-dire, par un quelconque outil produisant un "score" de réflexivité (certains auteurs ont tenté d'y parvenir, par exemple Kirby et Teddlie en 1989, mais ils ont abandonné leurs travaux). Aussi, son évaluation au sein de dispositifs de formation initiale doit être appréhendée par le biais de mesures « indirectes ", en l'occurrence, la recherche et l'analyse de traces écrites de réflexivité. Cette analyse, menée sur 16 dossiers réflexifs (14 étudiants des facultés de Psychologie et Sciences de l'Education et 2 de la faculté d'Economie et de Gestion), consiste à relever des indices textuels pouvant être associés à l'un des niveaux de réflexivité identifiés dans la littérature. II s'agit de répondre à la double question suivante : les étudiants ont-ils, à quelque degré que ce soit, fait preuve de réflexivité ? Si oui, à quel niveau de réflexivité cette manifestation peut-elle être associée?

\footnotetext{
${ }^{4} \mathrm{La}$ formation est divisée en quatre axes pour un total de 300 heures: les compétences socioculturelles, les connaissances pédagogiques, les compétences socio-affectives et relationnelles, le savoir-faire.
} 
Antoine Derobertmasure, Arnaud Dehon

Vers quelle évaluation de la réflexivité ?

\section{Méthodologie de recherche de « traces " de réflexivité écrite}

L'analyse de contenu, très utilisée dans le domaine des sciences humaines, consiste à "rechercher (dans un document) les informations qui s'y trouvent, dégager le sens ou les sens de ce qui y est présenté, formuler et classer tout ce que "contient " ce document" (Mucchielli, 2006, 24) en évitant le recours à l'intuition et en éliminant la subjectivité. L'analyse de contenu doit être objective, exhaustive, méthodique et quantitative. Elle s'intéresse au signifié de la langue (le sens de ce qui est produit), tandis que le signifiant (le véhicule du signifié) sera davantage un objet de recherche purement linguistique. Le syntagme " analyse de contenu " renvoie à bon nombre de méthodes différentes, issues de courants différents et aboutissant à des résultats spécifiques. Pour réaliser l'analyse des dossiers réflexifs remis par les enseignants en formation initiale, une méthodologie alternant deux types d'analyses de contenu a été élaborée : elle repose sur l'utilisation conjointe de deux analyses de contenu "outillées ", c'est-à-dire, recourant à l'utilisation d'un logiciel. L'une des analyses est une analyse propositionnelle ; elle a été menée en utilisant le logiciel Tropes. La seconde est une analyse de contenu thématique réalisée à l'aide du logiciel Nvivo. Ces deux méthodes (ainsi que les outils utilisés) sont présentées de manière plus approfondie dans les paragraphes suivants.

\section{L'analyse thématique}

L'analyse thématique consiste "à lire un corpus, fragment par fragment, pour en définir le contenu en le codant " (Fallery \& Rodhain, 2007, 20); il s'agit d'une méthode de décontextualisation - recontextualisation (Mukamurera, Lacourse \& Couturier, 2006; Deschenaux, 2007). Dans le cas de cette étude, la logique de travail a été délibérative puisque la grille de codage utilisée a été construite sur la base de plusieurs modèles ${ }^{5}$ de la réflexivité.

\section{Des modèles de la réflexivité...}

Le premier modèle, celui de Van Manen (1977), est construit sur la base de niveaux hiérarchiques. La réflexivité est donc appréhendée en termes de paliers allant d'une réflexion pragmatique (" technical reflection ") à une réflexion praxique (" pratical reflection ») jusqu'à une réflexion critique (" critical reflexion »). Ces étapes se distinguent en fonction de l'objet sur lequel porte la réflexion. Ainsi, le premier palier s'attache à un objet directement ancré dans l'action, à savoir la recherche de moyens afin de répondre efficacement et avec efficience ${ }^{6}$ aux objectifs fixés. Le deuxième palier - qui se distingue du premier par un certain détachement et une réflexion qui se décentre peu à peu de l'action même - porte sur les buts éducatifs fixés et s'explique comme un « engagement personnel avec pour but de développer une pratique personnalisée en fonction de croyances, de convictions et de valeurs personnelles " (Peters et al., 2005, 3). Finalement, au troisième niveau - niveau davantage macroscopique - la réflexivité porte sur le statut même d'enseignant et sur l'influence de la profession. Cette fois, la réflexion est davantage éthique, sociopolitique.

${ }^{5}$ Van Manen (1977); Sparks-Langer, Simmons, Pasch, Colton et Starko (1990); Hatton et Smith (1995) et Jorro (2005).

6 Pour les notions d'efficacité et d'efficience voir Demeuse, Baye, Straeten, Nicaise \& Matoul (2005). 
Evaluer les enseignants et les formateurs

Comment, pourquoi, pour quoi ?

Hatton et Smith (1995) ont adapté le modèle de Van Manen en spécifiant les quatre niveaux de réflexivité suivants : "descriptive writing " (description basique de ses actions), " descriptive reflection " (description avec justification des ses actions), "dialogic reflection " (jugement des événements, justification des choix réalisés et proposition d'alternatives) et "critical reflection" (recontextualisation de l'action par des connaissances historiques, sociopolitiques...).

Le troisième modèle étudié, également inspiré du modèle de Van Manen (1977), est celui de Sparks-Langer, Simmons, Pasch, Colton et Starko (1990). Ce modèle approfondit particulièrement la manière selon laquelle l'enseignant justifie son action ainsi que les termes auxquels il se réfère pour y parvenir. Ce modèle est élaboré en sept niveaux : 1) la simple narration des faits de classe, 2) la description des éléments de classe sans utilisation des termes adéquats, 3) la dénomination exacte des actions réalisées, 4) la justification des méthodes employées en réérence à des avis personnels et des habitudes de travail, 5) la justification des méthodes à l'aide d'arguments pédagogiques et théoriques, 6) la justification pédagogique soutenue par une justification en regard du contexte de la classe et enfin, 7) la justification basée sur les conceptions politiques, morales ou encore éthiques de l'Ecole de l'enseignant... Cette hiérarchie a fait l'objet de critiques dans la mesure où des (jeunes) enseignants pouvaient recourir à une réflexion d'ordre éthique sans pour autant avoir pu précédemment justifier leurs actions à l'aide de connaissances pédagogiques ou théoriques. Ces niveaux, comme ceux cités précédemment, doivent donc être pris avec certaines précautions (Hatton \& Smith, 1995).

Le dernier modèle, celui de Jorro (2005) est défini à partir de trois composantes, ellesmêmes subdivisées en plusieurs caractéristiques. Selon l'auteur, lorsqu'il s'agit d'analyser des écrits réflexifs, il est possible de se focaliser soit sur les seuils de réflexivité, soit sur les postures réflexives, soit sur les formes auto-évaluatives utilisées. Les seuils de réflexivité sont : le reflet (de type descriptif), l'interprétation (interrogation à partir d'un fait soulevé et remis en contexte) et la fonction critique régulatrice (proposition d'alternatives et prescriptions à adopter). Les différentes postures, basées sur les travaux de Fenstermacher et Richardson (1994), sont: le retranchement, le témoignage et le questionnement. Les formes d'autoévaluation sont : l'auto-évaluation et la tentation de l'idéal-type (un devenir), l'auto-évaluation troublée par l'idéal de soi (volonté de garder son image de soi identique), l'auto-évaluation assujettie aux transactions sociales (une forme de désirabilité sociale) et l'auto-évaluation annexée par la pensée narrative (réinterprétation des faits réels lors du passage à la narration).

La synthèse de ces modèles permet non seulement de lister les différentes formes de réflexivité documentées dans la littérature (la narration, la description et la justification de faits ; la proposition d'une alternative, la réflexion technique, l'exploration et l'évaluation d'une ou plusieurs alternatives, le questionnement d'une pratique en regard d'un modèle, la réflexion praxique et, finalement, la réflexion éthique) mais également d'appréhender la réflexivité selon divers angles d'approche. Ainsi, on peut se focaliser sur les objets sur lesquels porte la réflexivité de l'enseignant : de l'objet pragmatique (l'activité même) à l'objet éthique (valeurs, finalités, missions associés à l'éducation et/ou à l'Ecole...). II est également intéressant de prendre en compte les moments - phase préactive, phase interactive et phase postactive (Schön, 1994) - sur lesquels portent les propos de l'enseignant en formation initiale. Finalement, cette notion peut être abordée sous l'angle du processus développé par 
Antoine Derobertmasure, Arnaud Dehon

Vers quelle évaluation de la réflexivité ?

l'enseignant en formation lors de la rédaction d'un écrit réflexif; ce processus peut aller de la narration simple à la conceptualisation ou un étayage théorique (Sparks-Langer et al., 1990 ; Jorro, 2006).

\section{... à la grille de codage...}

La construction de la grille de codage nécessite l'articulation et l'intégration des quatre modèles de la réflexivité dans un modèle unique. II est pour cela nécessaire de recourir à un modèle permettant d'incorporer l'ensemble des différents aspects de la réflexivité. Les travaux de Vermersch (1994) concernant l'entretien d'explicitation et, plus précisément, les informations satellites de l'action, conviennent à cette fonction (fig.1).

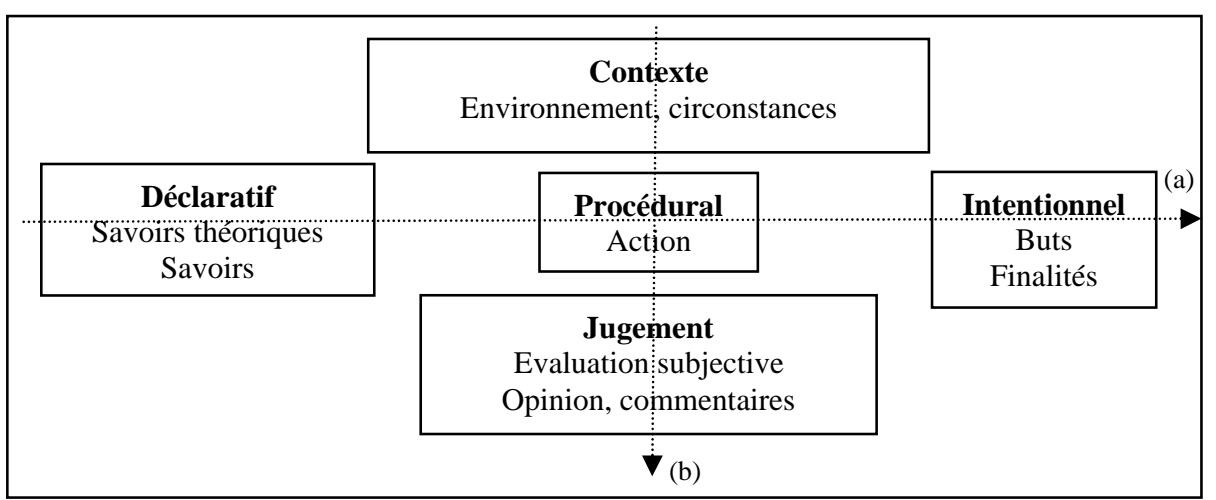

Fig. 1 - Les informations satellites de l'action (Vermersch, 1994)

Le modèle en question articule les propos que l'individu expose au sujet de l'action (aspect procédural) à ce que l'auteur nomme les satellites de l'action, c'est-à-dire, l'ensemble des informations ne concernant pas directement l'action mais s'attachant à des informations gravitant autour de l'action. II s'agit des informations concernant le contexte dans lequel s'est déroulée l'action, le jugement porté par l'enseignant en formation vis-à-vis de son action, les intentions poursuivies et, finalement, les connaissances et savoirs sur lesquels l'enseignant en formation initiale s'est basé pour agir.

A titre d'exemple, voici quelques illustrations concernant la mise en lien de différents modèles de la réflexivité à des informations satellites de Vermersch (op.cit.): le satellite "déclaratif " permet d'intégrer les différents niveaux de réflexivité du modèle de SparksLanger et ses collègues (1990); le satellite "intentionnel » permet de prendre en considération la technical reflection issue du modèle de Van Manen (1977) ainsi que la notion de finalité éthique de l'éducation relevée dans chacun des modèles ; le satellite " jugement " permet, quant à lui, d'intégrer la réflexion praxique également issue du modèle de Van Manen. 
Evaluer les enseignants et les formateurs

Comment, pourquoi, pour quoi ?

\section{...utilisée avec Nvivo}

Le logiciel utilisé pour réaliser cette analyse est le logiciel Nvivo. II appartient à la famille des CAQDAS7 et constitue "le choix le plus courant des qualitativistes recourant à l'informatique" (Lejeune, 2008, 594). Analysé du point de vue des typologies des logiciels d'analyse de contenu, il s'agit, selon la typologie de Jenny (1997) d'un logiciel appartenant au courant de l'analyse thématique/socio-sémantique tandis que du point de vue de la typologie de Lejeune (2008), il est qualifié d'outil de type réflexif, c'est-à-dire, sans automatisation de la démarche. Ce type de logiciel demande, de la part de l'analyste, une lecture exhaustive du corpus afin « de marquer (comme au "Stabilo " en mode manuel) et de coder les éléments textuels (sans contrainte de longueur ou de catégorie morpho-syntaxique) auxquels ils attribuent telle et/ou telle signification par rapport à la problématique de leur recherche " (Jenny, 1997, 91).

\section{L'analyse propositionnelle}

L'analyse propositionnelle "ne requiert aucun processus de codage, définit l'unité sémantique d'analyse et ne propose aucune catégorisation a priori. Tout texte est considéré comme constitué d'un ensemble de propositions; chacune d'elles représente l'unité sémantique d'analyse" (Ghiglione \& Blanchet, 1991, 105). Dans chaque proposition, un verbe relie des termes entre eux en leur attribuant une propriété. Pour plus de clarté, voici un exemple tiré de Ghiglione et Blanchet $(1991,106)$ : " "Tom mange une pomme dans la cuisine". II existe une relation de factivité entre l'actant "Tom" et l'acté "pomme" ; s'ajoute une relation avec un objet circonstant, en l'occurrence, "cuisine". Cette relation exprimée dans le discours se justifie, ici, par la notion de "micro univers" : un sujet traite une information en mettant en scène un ensemble structuré et plus ou moins cohérent de micro univers, chacun étant peuplé a minima d'un actant qui fait l'action et de l'acte que le verbe accomplit ».

\section{...et le logiciel Tropes}

Le logiciel utilisé pour cette analyse est le logiciel Tropes. II est basé sur une logique d'analyse sémantique de textes établie en six étapes de travail : le découpage ${ }^{8}$ du document en propositions (verbes, adjectifs, connecteurs...), la levée de l'ambiguité des mots du texte (homonymes par exemple), l'identification des classes d'équivalence sémantiques, la constitution de statistiques (rafales ${ }^{9}$ et épisodes ${ }^{10}$ ), la détection des contradictions du texte, et l'affichage des résultats. L'analyse sémantique réside notamment dans l'affectation des mots selon leur catégorie d'appartenance, dans l'étude de l'ordre d'arrivée des mots significatifs à l'intérieur d'une proposition et à l'intérieur du texte même, ce qui permet, notamment de dégager les styles discursifs utilisés par les locuteurs (Wolff \& Visser, 2005).

A partir des propositions, Tropes crée des univers "en regroupant les principaux substantifs du texte (noms communs et noms propres) grâce à des scénarios existants (dictionnaires d'équivalents sémantiques) et/ou à construire par le chercheur " (Fallery \&

\footnotetext{
${ }^{7}$ Acronyme qui signitie: Computer Assisted Qualitative Data Analysis Software. L'acronyme francophone ADQAO (Analyse de données qualitatives assistées par ordinateur) est beaucoup moins recensé dans la littérature scientifique.

${ }^{8}$ Fondé, notamment, sur les recherches de Benveniste (1974) et François (1986).

${ }^{9}$ Occurrence de mots.

10 Occurrence de rafales.
} 
Antoine Derobertmasure, Arnaud Dehon

Vers quelle évaluation de la réflexivité ?

Rodhain, 2007, 12). A partir de ces univers, divers types de relations peuvent être mises en évidence : univers juxtaposés dans les propositions, univers en position d'actant (c'est-à-dire, qui fait l'action) ou d'acté (c'est-à-dire qui subit l'action), univers davantage présents au début d'un texte ou à la fin ... Cette étape est permise grâce au dictionnaire sémantique du logiciel composé de réseaux d'équivalents sémantiques (Ghiglione et al, 1998). La configuration et l'utilisation de ce dictionnaire permettent d'analyser le texte à différents niveaux de généralité comprenant la catégorie des mots, des classes, des univers de référence 2 et des univers de référence 1.

\begin{tabular}{|l|l|l|l|l|}
\hline & \multicolumn{4}{|c|}{ Niveau de généralisation } \\
\hline & Mots & Classes & $\begin{array}{l}\text { Univers de } \\
\text { référence 2 }\end{array}$ & $\begin{array}{l}\text { Univers } \\
\text { référence 1 }\end{array}$ \\
\hline Exemple 1 & Réalisateur & Cinéma & Cinéma & Spectacle \\
\hline Exemple 1.b & $\begin{array}{l}\text { Équilibriste } \\
\text { Funambule }\end{array}$ & Acrobate & Cirque & Spectacle \\
\hline Exemple 2 & $\begin{array}{l}\text { Instituteur } \\
\text { Professeur }\end{array}$ & Enseignant & $\begin{array}{l}\text { École et } \\
\text { enseignement }\end{array}$ & $\begin{array}{l}\text { Enseignement et } \\
\text { éducation }\end{array}$ \\
\hline
\end{tabular}

Tab. 1 : exemple d'analyse selon le niveau de généralité (Tropes)

A la lecture du tableau 1, on constate que le mot « réalisateur » entre dans la classe " cinéma » qui s'emboite dans l'univers 2 " cinéma " lui-même emboité dans l'univers 1 "spectacle"; les mots "équilibriste » comme "funambule » entrent dans la classe "acrobate " emboitée dans l'univers 2 "cirque " lui-même inclus dans l'univers 1 "spectacle». Un autre exemple, lié au domaine de l'éducation, montre que les mots " instituteur » et « professeur » entrent dans la classe " enseignant » emboitée dans l'univers 2 "école et enseignement " lui-même compris dans l'univers 1 «enseignement et éducation $"$.

\section{Les résultats...}

\section{...de l'analyse thématique}

La prise en considération des quatre modèles de réflexivité retenus permet d'identifier 11 formes de réflexivité. La première question à laquelle l'analyse s'est intéressée a été de relever les différentes formes de réflexivité effectivement relevées dans les écrits réflexifs (voir tableau 2, page suivante).

On remarque dans ce tableau que certaines formes de réflexivité, bien que documentées dans la littérature, ne sont pas relevées par l'analyse de contenu des dossiers réflexifs. II s'agit par exemple de la réflexion éthique (qui est une forme de réflexivité commune aux quatre modèles retenus). Concernant l'absence de propos associés à la réflexion éthique, d'autres auteurs (Sparks-Langer et al. ; Hatton et Smith 1995) concluent également à cette quasi-absence de ce niveau de réflexivité en contexte de formation initiale d'enseignants. La littérature relative au développement des (jeunes) enseignants en cours de carrière (Fuller, 1969 ; Katz, 1972; Huberman, 1989) explique ce résultat de la manière suivante: la focalisation des jeunes enseignants sur les difficultés liées aux premières expériences de 
Evaluer les enseignants et les formateurs

Comment, pourquoi, pour quoi ?

classe constitue un obstacle à l'accès à une réflexion plus fondamentale de l'ordre de la réflexion éthique (Gervais, 1999).

\begin{tabular}{|c|c|c|c|}
\hline Formes de réflexivité & $\begin{array}{l}\text { Nombre } \\
\text { d'unités } \\
\text { codées }\end{array}$ & 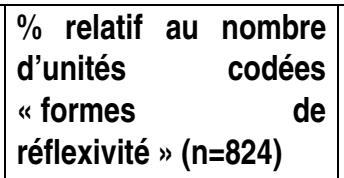 & $\begin{array}{l}\text { \% par rapport au } \\
\text { total } \quad \text { d'unités } \\
\text { codées }(n=1541)\end{array}$ \\
\hline Réflexion praxique & 336 & 40,78 & 21,8 \\
\hline Narration & 159 & 19,3 & 10,32 \\
\hline Description & 78 & 9,46 & 5,06 \\
\hline Réflexion technique & 70 & 8,6 & 4,5 \\
\hline Proposition d'une alternative & 69 & 8,37 & 4,48 \\
\hline Justification de la méthode & 63 & 7.55 & 4,08 \\
\hline Description théorique & 32 & 3,88 & 2,08 \\
\hline Exploration d'alternatives & 9 & 1,09 & 0,58 \\
\hline Evaluation d'une alternative & 8 & 0,97 & 0,52 \\
\hline Questionnement & 0 & 0 & 0 \\
\hline Réflexion éthique & 0 & 0 & 0 \\
\hline Total & 824 & 100 & 53,47 \\
\hline
\end{tabular}

Tab.2 : répartition des différentes formes de réflexivité

L'analyse de contenu s'est également intéressée à deux éléments importants de la réflexivité : il s'agit des objets de la réflexivité (à quoi l'étudiant fait-il référence dans ses propos : à son action, à celle de l'élève ou à la situation d'interaction entre lui et l'élève ?) et aux moments de la réflexivité (à quel moment de l'action l'étudiant fait-il référence dans ses propos : à la phase initiale, à la phase interactive ou la phase postactive ?). Le phénomène d'autocentration est également corroboré à ce niveau de l'analyse : l'objet des propos des enseignants en formation est essentiellement formulé en référence à leur propre action; la notion "d'élève » est quasiment évacuée de leur propos. En ce qui concerne le moment auquel les enseignants font référence, il s'agit principalement d'une focalisation sur la situation d'interaction (peu de références sont faites à la phase de planification, pourtant essentielle).

A l'inverse, la réflexion praxique est la forme de réflexivité la plus relevée dans les écrits réflexifs. Celle-ci, issue du modèle de Van Manen (1977) fait référence à l'évaluation que l'enseignant en formation initiale effectue. Cette évaluation peut porter sur trois aspects distincts : sa prestation dans le cadre du micro-enseignement (ex. : "j'étais inaudible »), les 
Antoine Derobertmasure, Arnaud Dehon

Vers quelle évaluation de la réflexivité ?

caractéristiques de la leçon (ex. : " l'alternance théorique pratique était bien structurée ") ou le comportement des élèves (ex. : "les élèves ne participaient pas beaucoup ").

Le tableau 3 présente les résultats relatifs à la catégorie des satellites de l'action.

\begin{tabular}{|l|r|r|r|}
\hline & unités codées & \% dans la catégorie & \% par rapport au total \\
\hline contexte & 230 & 35 & 14,93 \\
déclaratif & 40 & 6 & 2,60 \\
intentionnel & 47 & 8 & 3,05 \\
jugement & 336 & 51 & 21,80 \\
\hline Total & 653 & 100 & 42,38 \\
\hline
\end{tabular}

Tab. 3 - Unités codées dans la catégorie " satellites de l'information »

Des quatre sous-catégories constituant les satellites de l'action (tableau 3) deux d'entres elles regroupent $86 \%$ des unités de la catégorie : il s'agit des éléments relatifs au contexte (35\%) et au jugement (51\%). Cette dernière catégorie, " jugement », est celle qui a regroupé le plus d'unités codées ( $1 / 5$ des unités codées dans les dossiers réflexifs se rapportent à un jugement de l'enseignant en formation initiale. Ce résultat est déjà expliqué par la prédominance de la réflexivité de type praxique dans le tableau 2). Replacées sur la figure relative aux informations satellites de l'action (voir figure 1), ces deux catégories correspondent à l'axe (b) du modèle de Vermersch (1994) caractérisées par l'auteur comme concurrentes à l'explicitation de l'action tant ce type d'information est énoncé avec spontanéité par l'acteur. L'axe (a) (aspects déclaratifs et intentionnels) ne regroupe que $14 \%$ des unités codées. Cette information a un impact direct au point de vue de l'amélioration qui sera apportée au dispositif pédagogique. En effet, ce type de résultat, notamment au regard de la typologie de Sparks-Langer et ses collaborateurs (1990) peut interpeler. Selon ces auteurs, le moteur de la réflexivité, en formation initiale, se résume à pouvoir répondre à la question du "pourquoi ? " "We believe that asking the question "why" is crucial to the development of reflection in beginning teachers " (p.26) (la typologie de ces auteurs est d'ailleurs établie en ce sens). Aussi, dans l'optique des futures formations d'enseignants, le peu d'allusion à des éléments de cet ordre (cf. intentionnel) doit être pris en considération pour réguler le dispositif mis en place afin qu'il développe, en ce sens, la réflexion des enseignants en formation initiale.

...de l'analyse propositionnelle

Le tableau 4 présente cinq références les plus utilisées dans les 16 dossiers réflexifs :

\begin{tabular}{|l|r|r|r|}
\hline Références utilisées & Dans combien de dossiers? & Actant & Acté \\
\hline Enseignant & 16 & 15 & 1 \\
\hline Cours & 16 & 1 & 15 \\
\hline Élève & 16 & 0 & 16 \\
\hline Support pédagogique & 10 & 0 & 10 \\
\hline Enseignant et collègue & 6 & 3 & 3 \\
\hline
\end{tabular}

Tab. 4 : cinq références significativement présentes dans les dossiers réflexifs 
Evaluer les enseignants et les formateurs

Comment, pourquoi, pour quoi ?

Les deux dernières colonnes de ce tableau informent le lecteur au sujet de la dimension actant-acté. Ce type de données est relativement important car elles renseignent sur le sens des relations entre les références utilisées. Exprimé autrement, cela signifie que cette information permet de distinguer la ou les référence(s) "qui fait/font l'action ", c'est-à-dire l'actant, de la ou les référence(s) "qui subit/subissent l'action ", c'est-à-dire l'acté. Dans le cadre de cette recherche, on constate que l'enseignant occupe presque exclusivement la position d'actant, "celui qui fait les actions ", tandis que les élèves occupent toujours la position d'actés. Ce résultat, constitué d'une toute autre manière que ceux communiqués par l'analyse thématique, conforte que l'objet de la réflexion des enseignants est essentiellement leur propre action en évacuant la dimension élève de leur propos, du moins en tant qu'acteur.

Ce tableau apporte également deux informations que l'analyse thématique n'a pas révélées. Premièrement, il s'agit de l'importance que les enseignants en formation initiale ont attribuée, dans leur écrit réflexif, au travail en duo ${ }^{11}$. Les six enseignants en formation faisant significativement référence à leur collègue de travail (5ème ligne du tableau : " enseignant et collègue ") sont des individus ayant travaillé ensemble. La dimension " actant-acté " indique une différence quant à la conception de l'action du « duo »: dans 3 des 6 dossiers, le duo apparaît comme une référence effectuant l'action tandis que dans les trois autres, le duo reste en quelque sorte subordonné à l'action de l'individu pris de manière isolée. Dans le même ordre d'idée, les résultats proposés par le logiciel Tropes ont également permis d'identifier un obstacle particulièrement rencontré par les enseignants en formation initiale : il s'agit des difficultés relatives à l'élaboration et la gestion, en situation d'interaction, des outils pédagogiques (entendus au sens large du terme, c'est-à-dire, le tableau, les transparents, les documents audio-visuels, les feuilles-élèves, etc.). C'est clairement à ce type de résultat que font allusion Wolff et Visser $(2005,103)$ lorsqu'ils notent que « l'on peut découvrir des univers sémantiques insoupçonnés, car ne faisant initialement pas l'objet de questions particulières de la part de l'analyste, et ne faisant donc pas l'objet de questions particulières ".

Ce type d'information conforte l'importance attribuée par ces enseignants en formation au travail en duo, confirme le bien fondé de ce type d'association et ouvre de nouvelles perspectives de recherche concernant le développement de la réflexivité en formation initiale.

\section{Conclusions et perspectives}

Le recours à une double analyse de contenu a permis de mettre en avant certaines formes de réflexivité. Les principaux résultats indiquent que les enseignants en formation initiale ont principalement tendance à évaluer leur propre prestation durant la séance d'interaction ; les dimensions relatives à l'élève et à l'interaction avec celui-ci sont quasiment absentes des propos des futurs enseignants. On note également que les enseignants en formation initiale justifient très peu les choix pédagogiques opérés en phase de préparation de leçon (planification) et lors de la situation d'interaction. Aussi, ce type de résultat revient à s'interroger sur la manière d'amener le développement des compétences réflexives en contexte de formation initiale. En effet, comment en tant que formateur, pousser les enseignants en formation initiale à une écriture réflexive sans que celle-ci ne corresponde à

\footnotetext{
${ }_{11}$ Dans le cadre du micro-enseignement, les étudiants devaient préparer, par 2, deux préparations de leçon.
} 
Antoine Derobertmasure, Arnaud Dehon

Vers quelle évaluation de la réflexivité ?

une stricte réponse à une consigne, laquelle pouvant alors même apparaître comme une injonction paradoxale du type "sois réflexif "?

Une autre interrogation concerne le rapport entre ce que le formateur connait des modèles de réflexivité et l'orientation de l'écriture réflexive. Dit autrement, si le formateur se réfère, par exemple, principalement au modèle de Sparks-Langer et ses collègues (basé sur la justification de l'action), doit-il, dans les consignes de rédaction, explicitement demander aux enseignants en formation initiale de légitimer leur action ${ }^{12}$ ? Dans ce cas, non seulement la première question concernant le lien entre consigne et écriture est reposée mais en plus, la moindre analyse de contenu menée sur les dossiers réflexifs ainsi constitués ne pourraient que conclure à un respect ou non des consignes de travail et non à la manifestation de formes de réflexivité. Une manière de solutionner ce problème réside, peut-être ${ }^{13}$, dans l'adjonction d'une séance supplémentaire au dispositif pédagogique. Lors de celle-ci, l'enseignant en formation initiale et le superviseur, sur la base du visionnement de la prestation enregistrée sur DVD, interagissent. Cette technique de video stimulated recall (Schepens, Aelterman, Van Keer, 2007), repose sur l'articulation de deux temps : mise en situation/enregistrement et visionnement/analyse conjointe. Cette méthode est usitée dans les contextes où le think aloud protocol ne peut être utilisé pour des raisons contextuelles et/ou dans des situations où la verbalisation de l'action " en situation " pourrait affecter l'efficacité de l'action. C'est le cas pour les enseignants (Stough, 2001). D'un point de vue pédagogique, cette séance permet d'amener l'enseignant en formation initiale à s'exprimer oralement au sujet de sa prestation (ce qui constitue un enrichissement par rapport à la seule trace écrite de réflexivité). De plus, lors de cet échange, le superviseur peut amener l'enseignant en formation à se prononcer quant à certains aspects de la réflexivité jugés importants, notamment, la justification de l'action.

D'un point de vue de la recherche, ces entretiens enregistrés constituent un matériau d'analyse intéressant: ils permettent premièrement de mettre à jour le type de réflexivité propre à l'interaction entre l'enseignant en formation et le superviseur; deuxièmement, de distinguer la part de prise de parole spontanée de l'enseignant en formation versus la justification "provoquée ", c'est-à-dire, les réponses apportées suite à une sollicitation du superviseur; troisièmement, l'analyse de la nature des interactions établies entre le superviseur (telles que: poser une question, demander une explication, demander une reformulation, énoncer un argument contraire...) et le supervisé (telles que: décrire la situation, évaluer sa prestation, apporter une justification...) mettra à jour les mécanismes sous-jacents de la supervision opérée au départ du visionnement du DVD. Cette opération permettra à son tour l'identification des interventions du superviseur jugées pertinentes (c'està-dire, amenant davantage l'enseignant en formation initiale à interroger sa pratique). In fine, par l'amélioration de la phase de supervision, c'est l'amélioration générale du dispositif qui est visée.

L'analyse propositionnelle a souligné l'importance du travail en binôme (relevée par plus d'un tiers des enseignants en formation initiale ayant participé à notre dispositif). Etant donné que cette dimension ne faisait pas initialement l'objet de notre recherche, aucune prise

\footnotetext{
12 A ce sujet Sparks-Langer et ses collègues (1990) recommandent d'orienter davantage les écrits réflexifs en fonction du modèle réflexif sur la base duquel la formation est organisée.

${ }^{13}$ Cette question fait actuellement l'objet de recherches.
} 


\section{Evaluer les enseignants et les formateurs \\ Comment, pourquoi, pour quoi ?}

d'information concernant la manière de procéder des groupes n'a été organisée. A l'avenir, cette prise d'information permettra, d'une part, de caractériser le processus du travail en duo dans le cadre de la formation initiale et, d'autre part, d'identifier les particularités (mode de constitution, mode de répartition du travail, processus de travail...) des paires d'enseignants en formation fonctionnant mieux que les autres.

Finalement, la particularité de cette recherche concerne la méthodologie mise en place en ce sens que deux logiciels d'analyse de contenu ont été utilisés. Ce choix, justifié par la complémentarité existant entre les deux méthodes, renvoie à une remarque générale concernant la notion d'outil et le rapport qu'il est nécessaire d'entretenir avec celle-ci. Comme le signale Aubert (2000), le recours à un logiciel informatique pour analyser un corpus écrit ne doit pas aboutir à un aveuglement technique ou encore, selon Weitzman (2003), à la certitude selon laquelle le recours à un logiciel signifie rigueur méthodologique. Aubert (2000, 33), reprenant les propos de Bardin (1980), rappelle d'une part, qu' « on obtiendra à la sortie que ce qui a été mis à l'entrée, le mauvais comme le bon, l'inutile comme l'utile" et, d'autre part, que les indices numériques fournis par le logiciel ne peuvent permettre l'économie d'un retour au texte et d'une " lecture flottante " de celui-ci. II s'agit d'un travail délicat soumis à la subjectivité et l'interprétation de l'analyste (Trudel, 1999) lequel devra faire preuve de rigueur méthodologique et de transparence, c'est-à-dire, assurer une forme de traçabilité du " chemin » parcouru depuis l'acquisition des données jusqu'à la proposition de résultats.

\section{Bibliographie}

Amigues, R., Faïta, D. \& Saujat, F. (2004). L'autoconfrontation croisée : une méthode pour analyser l'activité enseignante et susciter le développement de l'expérience professionnelle. Bulletin de psychologie, 469, 41-44.

Aubert, A. (2000). Valeurs, validité, valence de l'expérience professionnelle et activités du conseil. Toulouse : thèse doctorale (non encore publiée).

Bardin, L. (2001). L'analyse de contenu. Paris: Presses Universitaires de France.

Boutet, M. (2004). La pratique réflexive: un apprentissage à partir de ses pratiques. Conférence au ministère de l'Éducation, des Loisirs et des Sports du Québec. En ligne : [http://www.mels.gouv.qc.ca/REFORME/rencontres.htm] (page consultée le 25 mars 2008). De Cock, G. (2007). Le journal de bord, support de la réflexion sur la pratique professionnelle pour les futurs enseignants en stage. Louvain-la-Neuve : Thèse doctorale.

Demeuse M., Baye A., Straeten M-H., Nicaise J., Matoul, A. (2005). Vers une école juste et efficace. 26 contributions à l'analyse des systèmes d'enseignement et de formation. Bruxelles : De Boeck Université.

Deschenaux, F. (2007). Guide d'introduction au logiciel QSR Nvivo 7. Trois-Rivières. En ligne : [http://www.recherche-qualitative.qc.ca/Nvivo7.pdf] (page consultée le 25 mars 2008) Donnay, J. \& Charlier, E. (2006). Apprendre par l'analyse des pratiques: initiation au compagnonnage réflexif. Namur : Presses Universitaires de Namur.

Durand, M. (1996). L'enseignement en milieu scolaire. Paris: Presses universitaires de France.

Faïta, D. \& Vieira, M. (2003). Réflexions méthodologiques sur l'autoconfrontation croisée. Skolhê, 1, 57-68. 
Fallery, B. \& Rodhain, F. (2007). Quatre approches pour l'analyse de données textuelles: lexicale, linguistique, cognitive, thématique. Montréal: 16ème Conférence Internationale de Management Stratégique. En ligne : [http://www.strategie-aims.com/aims07/www.aims2007. uqam.ca/actes-de-la-conference/communications/falleryb300/at_download/article.pdf] (page consultée le 15 février 2008).

Fenstermacher, G. \& Richardson, V. (1994). L'explicitation et la reconstruction des arguments pratiques dans l'enseignement. Cahiers de la recherche en éducation, 1(1), 157-182.

Fuller, F.F. (1969). Concerns of teachers: a developmental conceptualization. American Educational Research Journal, 6(2), 207-226.

Katz, L. (1972). Developmental stages of preschool teachers. Elementary School Journal, 73(50), 123-127.

Gervais, C. (1999). Analyse du discours d'enseignants sur des pratiques d'accompagnement en insertion professionnelle: une pratique d'accompagnement à l'image de la pratique d'enseignement. In J.-C. Hétu, M. Lavoie \& S. Baillauquès (Ed.), Jeunes enseignants et insertion professionnelle: un processus de socialisation? de professionnalisation? de transformation ? Bruxelles: De Boeck, 113-138.

Gervais, C. \& Lepage, M. (2000). Transfert de la responsabilité de l'évaluation en stage du superviseur vers l'enseignant associé : un pas de plus vers la professionnalisation. In D. Martin, C. Garant, C. Gervais, C. Saint-Jarre (Ed.), Recherches et pratiques en formation des maîtres. Montréal : Editions du CRP, 113-128.

Ghiglione, R. \& Blanchet, A. (1991). Analyse de contenu et contenus d'analyse. Paris: Dunod.

Ghiglione, R., Landré, A., Bromberg, M. \& Molette, P. (1998). L'analyse automatique des contenus. Paris : Dunod.

Hatton, N. \& Smith, D. (1995). Reflection in teacher education: towards definition and implementation. Teaching and Teacher Education, 11(1), 33-49.

Huberman, M. (1989). La vie des enseignants. Évolution et bilan d'une profession. Neuchâtel: Delachaux et Niestle.

Jenny, J. (1997). Méthodes et pratiques formalisées d'analyse de contenu et de discours dans la recherche sociologique française contemporaine. Etat des lieux et essai de classification. Bulletin de Méthodologie Sociologique, 54, 64-112.

Jorro, A. (2005). Réflexivité et auto-évaluation dans les pratiques enseignantes. Mesure et évaluation en éducation, 27(2), 33-47.

Jorro, A. (2006). Devenir ami critique. Avec quelles compétences et quels gestes professionnels? Mesure et évaluation en éducation, 29(1), 31-44.

Kirby, P.C. \& Teddlie, C. (1989). Development of the reflective teaching instrument. Journal of Research and Development in Education, 22(4), 45-51.

Kirby, P. C., \& Paradise, L. V. (1992). Reflective practice and effectiveness of teachers. Psychological Reports, 70, 1057-1058.

Lang, V. (1996). Professionnalisation des enseignants, conceptions du métier, modèles de formation. Recherche et formation, 23, 9-27.

Lejeune, C. (2008). Au fil de l'inteprétation - L'apport des registres aux logiciels d'analyse qualitative. Swiss Journal of Sociology, 34(3), 593-603.

Maroy, C. (2001). Le modèle du praticien réflexif à l'épreuve de l'enquête. Cahier de recherche du Girsef, 12. 
Evaluer les enseignants et les formateurs

Comment, pourquoi, pour quoi ?

Martin, D., Garant, C., Gervais, C., \& Saint-Jarre, C. (2000). Recherches et pratiques en formation des maîtres. Montréal : Editions du CRP.

Maubant, P. (2007a). Sens et usages de l'analyse des pratiques d'enseignement: entre conseil et accompagnement réflexif des enseignants en formation. Les dossiers des Sciences de l'Education, 18, 39-48.

Mucchielli, R. (2006). L'analyse de contenu des documents et des communications. Issy-LesMoulineaux : ESF (gème édition).

Mukamurera, J., Lacourse, F. \& Couturier, Y. (2006). Des avancées en analyse qualitative : pour une transparence et une systématisation des pratiques. Recherches qualitatives, 26(1), 110-138.

Paquay, L. (2005). Quelle formation des enseignants en Belgique francophone? Formation et Profession, 11(1), 28-34.

Paquay, L., Altet, M., Charlier, E. \& Perrenoud, P. (2006). Former des enseignants professionnels : quelles stratégies? Quelles compétences ? Bruxelles : De Boeck.

Paquay, L. \& Wagner, M.-C. (2001). Compétences professionnelles privilégiées dans les stages et en vidéo-formation. In L. Paquay, M. Altet, E. Charlier \& Ph. Perrenoud (Ed.). (2001). Former des enseignants professionnels. Quelles stratégies? Quelles compétences? Bruxelles : De Boeck, 76-101.

Perrenoud, P. (2001). Mettre la pratique réflexive au centre du projet de formation. Cahiers pédagogiques, 390, 42-45.

Peters, M., Chevrier, J., Leblanc, R., Fortin, G. \& Malette, J. (2005). Compétence réflexive, carte conceptuelle et webfolio à la formation des maîtres. Canadian Journal of Learning and Technology, 31(3)

Scheepers C. (2005). Comment évaluer la réflexivité ? Outils, dispositifs et acteurs. Reims : Actes du 18ème colloque ADMEE.

Schepens, A., Aelterman, A. et Van Keer, H. (2007). Studying learning processes of student teachers with stimulated recall interviews through changes in interactive cognitions. Teaching and teacher education, 23, 457-472.

Schön, D. (1983). The Reflective Practitioner. New York : Basic Books

Schön, D. (1994). Le praticien réflexif. A la recherche du savoir caché dans l'agir professionnel. Montréal : Les éditions Logiques.

Sparks-Langer, G. M., Simmons, J. M., Pasch, M., Colton, A., \& Starko, A. (1990). Reflective pedagogical thinking: How can we promote it and measure it? Journal of Teacher Education, 41(5), 23-32.

Stough, L. (2001). Using stimulated recall in classroom observation and professional development. Actes de Annual meeting of the American Educational Research Association, Seattle.

Tochon, F.V. (1993). L'enseignant expert. Paris : Nathan.

Trudel, P. (1999). Compléter la formation des chercheurs avec le logiciel NUD.IST. Recherches qualitatives, 20, 87-111.

Van Manen, M. (1977). Linking ways of knowing with ways of being practical. Curriculum Inquiry, 6, 205-228.

Vermersch, P. (1994). L'entretien d'explicitation en formation initiale et en formation continue. Paris : ESF.

Wagner, M-C. (1998). Pratique du micro-enseignement. Bruxelles : De Boeck. 
Antoine Derobertmasure, Arnaud Dehon

Vers quelle évaluation de la réflexivité ?

Wittorski, R. (2007). Professionnalisation et développement professionnel. Paris: L'Harmattan.

Wolff, M., \& Visser, W. (2005). Méthodes et outils pour l'analyse des verbalisations : une contribution à l'analyse du modèle de l'interlocuteur dans la description d'itinéraires. @ctivités, 2(1).

Yvon, F. \& Garon, R. (2006). Une forme d'analyse du travail pour développer et connaître le travail enseignant : l'autoconfrontation croisée. Recherches qualitatives, 26, 51-80. 